\title{
Bias-Dependent Charge Accumulation in Pentacene-based Thin-Film Transistors
}

\author{
Chi-Feng Lin ${ }^{a}$, Kai-Hsiang Chuang ${ }^{\text {a }}$, Yet-Min Chen ${ }^{\text {a }}$, Jiun-Haw Lee ${ }^{\text {*a }}$, Jian-Jang Huang a , \\ and $\mathrm{Yu}-\mathrm{Wu}$ Wang ${ }^{\mathrm{b}}$ \\ ${ }^{a}$ Graduate Institute of Electro-Optical Engineering and Department of Electrical Engineering, \\ National Taiwan University, No. 1, Sec. 4, Roosevelt Road, Taipei, Taiwan, R.O.C. \\ ${ }^{b}$ Electronics Research \& Service Organization, Industrial Technology Research Institute, Hsinchu, \\ Taiwan 310, ROC
}

\begin{abstract}
In this paper, we have demonstrated the current increase with repeated measurements of $I_{d}-V_{d s}$ curves with different $V_{g}$ values which results from the non-uniform carrier accumulation in the channel region of a pentacene-based thin film transistor (TFT). The mobility of our device reaches $0.07 \mathrm{~cm}^{2} / \mathrm{Vs}$ even the substrate was not heated during pentacene deposition. Besides, the devices show good air-stable properties. The magnitude of $I_{d}$ decreased less than $30 \%$ after exposure in air for 2 weeks. By repeating the $\mathrm{I}_{\mathrm{d}}-\mathrm{V}_{\mathrm{ds}}$ measurements from 0 to $-50 \mathrm{~V}$ with the $\mathrm{V}_{\mathrm{g}}$ values of $0,-10,-20,-30$, -40 , and $-50 \mathrm{~V}$ for 10 minutes, we observed a four times current increase from -0.75 to $-2.8 \mu \mathrm{A}$ at $\mathrm{V}_{\mathrm{g}}=-50 \mathrm{~V}$ and $\mathrm{V}_{\mathrm{ds}}=-$ $50 \mathrm{~V}$. The current increase comes from the holes accumulation near the drain. When the source and drain were exchanged, the current decreases to the $0.08 \mu \mathrm{A}$. After another 10 minutes operation, the current will recover back to the original values. Such a process is reversible and shows the potential of the memory device base on this pentacene transistor.
\end{abstract}

Keywords: organic thin-film transistor, mobility, carrier transport

\section{INTRODUCTION}

Owing to the advantages like low-cost processing, large-area and flexible-substrate capability, organic thin-film transistors (OTFTs) have recently attracted much attention [1], [2]. Recently, carrier mobility of OTFT has a great improvement. Among them, pentacene is one of the most successful organic materials used for the channel layer of $\mathrm{p}$ type OTFTs due to its high hole-mobility [3]. Typically, the organic layers are thermally evaporated onto the substrate. In order to enhance the performance by better aligning the organic molecule, the substrate is usually heated during the evaporation [4]. However, it increases the cost and the complexity of the process. In this work, we demonstrate a pentacene based OTFT with the high mobility and air-stable characteristic without heating the substrate during the evaporation process and discuss the carrier accumulation in the channel influence from the voltage bias [5]. In this paper, the experiment details are given in section 2. Experimental results and the related discussions are presented in section 3. Finally, a summary is drawn in section 4.

\section{EXPERIMENT}

Fig. 1 shows the configuration of our OTFT device, we use a bottom gate and bottom contact structure. The electrodes, including source, drain, and gate are all made by indium-tin-oxide (ITO) with a thickness of $100 \mathrm{~nm}$, which were deposited on a glass substrate by sputtering. The material of gate insulator is tetraethoxysilane (TEOS) with a thickness of $300 \mathrm{~nm}$. It was prepared by plasma-enhanced chemical vapor deposition (PECVD). The channel lengths of the devices were 17, 27, 37, 47, and $57 \mu \mathrm{m}$, and the channel width were all $500 \mu \mathrm{m}$.

*jhlee@cc.ee.ntu.edu.tw; phone 8862 2363-5251 ext. 540; fax 8862 2367-7467

Organic Field-Effect Transistors V, edited by Zhenan Bao, David J. Gundlach

Proc. of SPIE Vol. 6336, 63361F, (2006) · 0277-786X/06/\$15 · doi: 10.1117/12.678972

Proc. of SPIE Vol. $633663361 \mathrm{~F}-1$ 
The substrate was first cleaned by supersonic bath with de-ionized water, acetone and methanol, each for 5 minutes. Then the substrate was baked at $130{ }^{\circ} \mathrm{C}$ for 20 minutes by a hot-plate and treated by oxygen plasma for 90 sec. After all the pretreatment, the pentacene active layer was deposited by thermal evaporation with the deposition rate of $0.4 \AA / \mathrm{sec}$ and the thickness of $1000 \AA$ through a steel shadow mask. Without heating the substrate, the temperature of the substrate was kept in room temperature and the pressure of the chamber was kept in high vacuum of $6^{*} 10^{-6}$ during the evaporating. After the evaporation, an HP 4156C Precision Semiconductor Parameter Analyzer was used to measure the characteristic of the OTFTs. The OTFTs were all measured and stored under air environment.

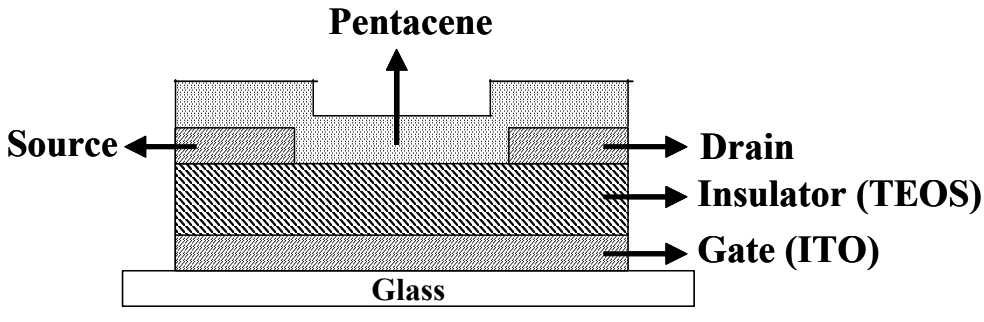

(a)

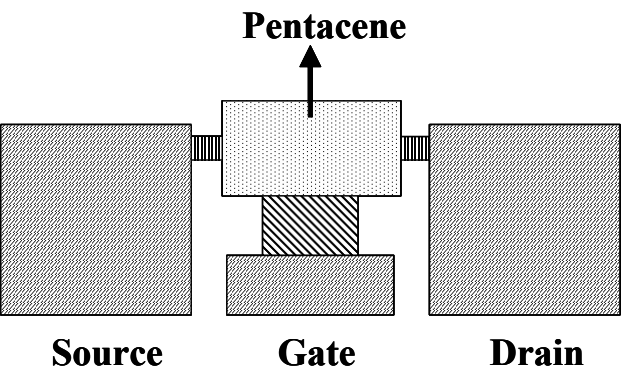

(b)

Fig. 1 Device structure of the OTFT. (a) Cross section (b) top view

\section{RESULTS AND DISCUSSIONS}

Fig. 2 (a) and (b) shows the $I_{D}-V_{D S}$ curves for the OTFT without and with baking after the wet clean processes of a channel length of $57 \mu \mathrm{m}$ with varying $\mathrm{V}_{\mathrm{G}}$ values from $0,-10,-20,-30,-40$ and $-50 \mathrm{~V}$, respectively. From Fig. 2(a), it can be observed that the leakage current is very large when the substrate is not baked. On the other hand, after baking at 130

for $20 \mathrm{~min}$ (Fig. 2 (b)), we can see the leakage current has a dramatic decrease. Hence, we assume that the leakage current is attributed to the solvent which affect the carrier transfer in the channel or direct affect by the mobile ion from the solvent.

The mobility values of the OTFT can be deduced from the basic transistor equations

$$
\begin{aligned}
& I=\mu C_{o x} \frac{W}{L}\left[\left(V_{g}-V_{t}\right) V_{d s}-\frac{1}{2} V_{d s}^{2}\right]+I_{d a r k} \\
& I=\frac{1}{2} \mu C_{o x} \frac{W}{L}\left(V_{g}-V_{t}\right)^{2}+I_{d a r k}
\end{aligned}
$$

Eq. 1 and 2 describe the I-V characteristics in the triode and saturation region, respectively. In these equations, $\mathrm{W}$ is the channel width, $\mathrm{L}$ is the channel length, $\mu$ is the mobility, $\mathrm{V}_{t}$ is the threshold voltage which can be obtained from the curve of drain current versus gate voltage $\left(\mathrm{I}_{\mathrm{d}}-\mathrm{V}_{\mathrm{g}}\right), \mathrm{C}_{\mathrm{ox}}$ is the capacitance per unit area of the insulating layer, and $\mathrm{I}_{\text {dark }}$ is the dark current or leakage current. From Fig. 2 (b), we can derive that the mobility value is $0.07 \mathrm{~cm}^{2} / \mathrm{Vs}$ in our devices and the $\mathrm{I}_{\mathrm{d}}=12 \mu \mathrm{A}$ under the operation with $\mathrm{V}_{\mathrm{g}}=-50 \mathrm{~V}$ and $\mathrm{V}_{\mathrm{ds}}=-80 \mathrm{~V}$, which is quite high for the OTFTs fabrication without heating the substrate. It may result from oxygen plasma treatment and/or other surface treatment before the evaporation. 


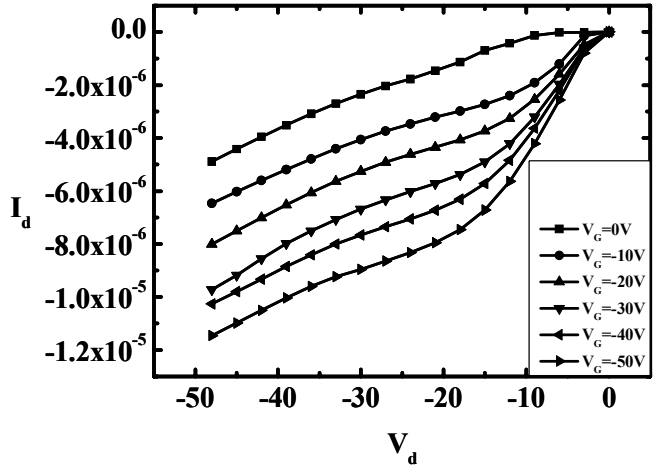

(a)

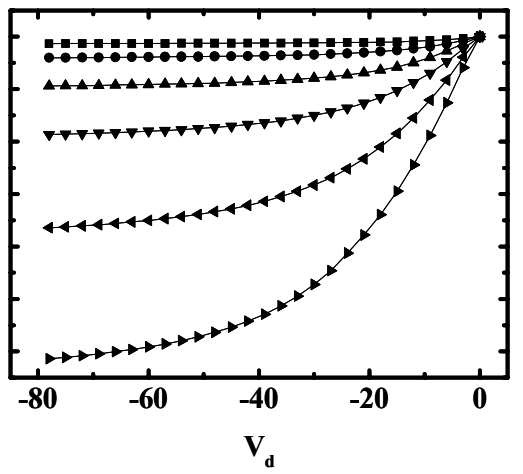

(b)

Fig. $2 \mathrm{I}_{\mathrm{d}}-\mathrm{V}_{\mathrm{ds}}$ curves of the OTFT. (a) Without baking the substrate before the evaporation. (b) The substrate was baked at 130 for 20 minutes before the evaporation.

Fig. 3 shows the $I_{d}-V_{d s}$ curves for the OTFT measured at different storage time. During the storage, the OTFT are put in the air environment and there is no passivation layer on the devices.

Compared the drain current value after 3 days and 12 days storage, the drain current of the OTFT with the channel length of $37 \mu \mathrm{m}$ was slightly decreased from $-0.63 \mu \mathrm{A}$ to the value of $-0.56 \mu \mathrm{A}$ in 9 days, under the operation with $\mathrm{V}_{\mathrm{g}}=-$ $50 \mathrm{~V}$ and $\mathrm{V}_{\mathrm{ds}}=-50 \mathrm{~V}$. And for the device with the channel length of $57 \mu \mathrm{m}$, the drain current was decreased from -0.52 $\mu \mathrm{A}$ to the value of $-0.44 \mu \mathrm{A}$. It shows the well air-stable properties for the magnitude of the drain current decreased less than $30 \%$ compared to the initial value after exposure in air for about 2 weeks.
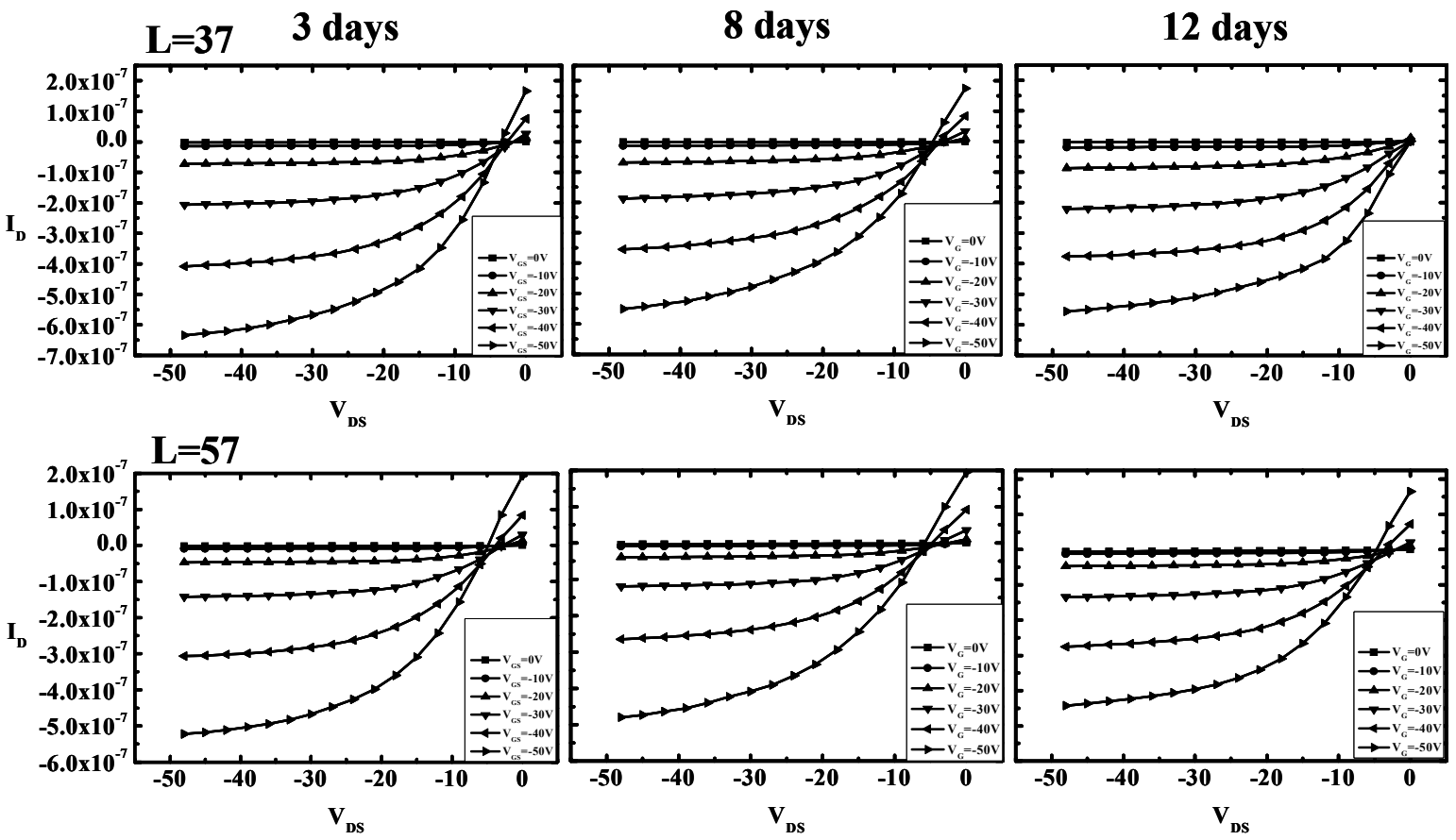

Fig. $3 \mathrm{I}_{\mathrm{d}}-\mathrm{V}_{\mathrm{ds}}$ curves of the OTFT with different storage time. 
To discuss the behavior of the carrier accumulation in the channel under the bias stress, a DC bias stress experiment was conducted. Fig. 4 shows the drain current versus time with $\mathrm{V}_{\mathrm{DS}}=-35 \mathrm{~V}, \mathrm{~V}_{\mathrm{G}}=-50 \mathrm{~V}$ and the corresponding $\mathrm{I}_{\mathrm{D}}-\mathrm{V}_{\mathrm{DS}}$ curves of different stressing time. In Fig. 4(a), the current was increased dramatically from $-0.39 \mu \mathrm{A}$ to $-1.8 \mu \mathrm{A}$ at the first 20 minutes and then was nearly maintained around $-1.6 \mu \mathrm{A}$ for the remaining 3 hours. The same behavior was also observed in the corresponding $\mathrm{I}_{\mathrm{d}^{-}}-\mathrm{V}_{\mathrm{ds}}$ curves of different stressing time in Fig. 4(b), the drain current was increased from $-0.27 \mu \mathrm{A}$ before the bias stress to $-0.46 \mu \mathrm{A}$ after 3 hours stressing operation, and recovered to $-0.25 \mu \mathrm{A}$ after the bias stress for 1 day. It shows a significant enhancement after the bias operation. In the case of largest enhancement, the later current was nearly 2 times of the original value and then recovered to almost the same value as the current before bias operation after removing the bias voltage. This current enhancement effect is reproducible. Once again we apply the bias, the current enhancement will be observed again. It indicates that the influences on the current caused by the voltage bias are reversible and reproducible. The origins of these effects may come from the nonuniformly accumulation of the carriers in the channel under the voltage bias [6].

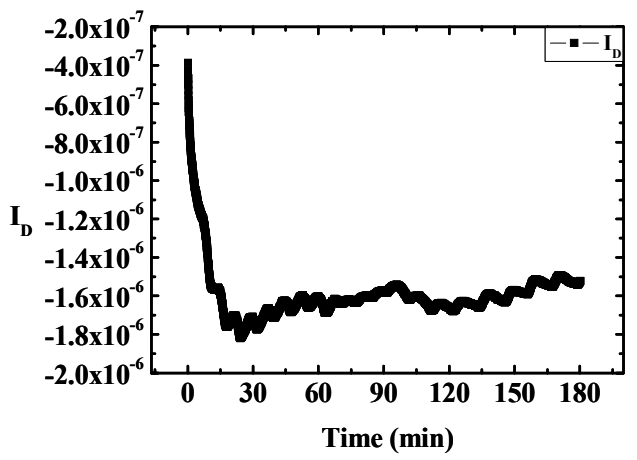

(a)

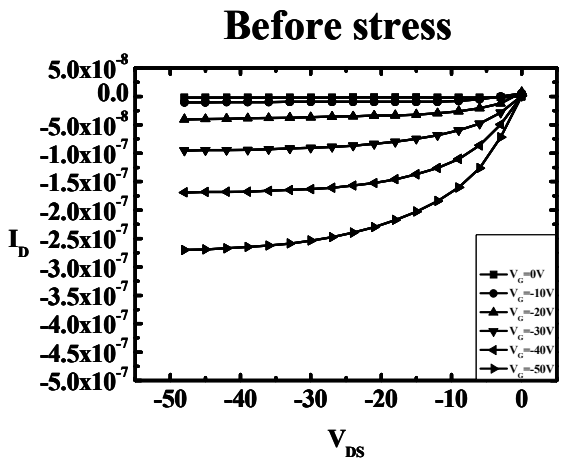

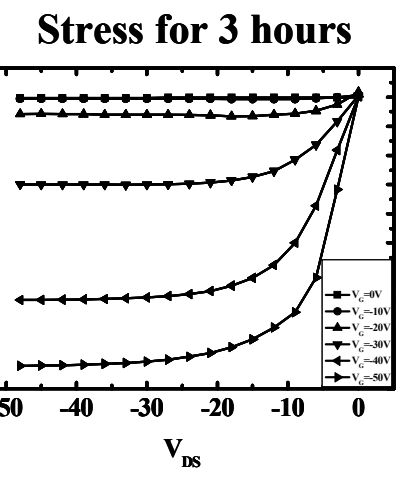

(b)
1 day after stress for $10 \mathrm{hr}$

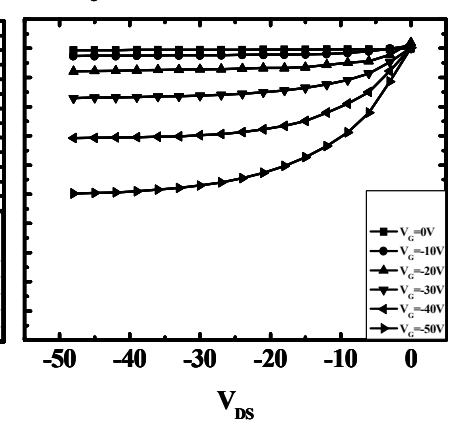

Fig. 4 (a) $I_{D}$ versus time under DC stress. (b) $I_{d}-V_{d s}$ curves of the corresponding stressing time.

Fig. 5 shows another result of the bias stress operation with $\mathrm{V}_{\mathrm{ds}}=-50 \mathrm{~V}, \mathrm{~V}_{\mathrm{g}}=-50 \mathrm{~V}$ for 24 hours and the corresponding $\mathrm{I}_{\mathrm{d}}-\mathrm{V}_{\mathrm{ds}}$ curves after different stressing time. In this case, the current was increased from $-11 \mu \mathrm{A}$ to -13.4 $\mu \mathrm{A}$ during the first 10 minutes bias operation and was decreased to $-3.6 \mu \mathrm{A}$ in 24 hours. It shows the current was still increased under higher voltage bias, but it was decreased rapidly during the remaining operation. The same result can also be observed from the drain current values in the $I_{D}-V_{D S}$ curves, under the operation with $V_{g}=-50 \mathrm{~V}$ and $V_{d s}=-80 \mathrm{~V}$, the drain current value was increased from $-14.1 \mu \mathrm{A}$ to $-20 \mu \mathrm{A}$ and then was decreased to $-0.78 \mu \mathrm{A}$. This result shows that the drain current under the bias stress is related to the operation voltage. If the bias voltage is too large, the performances of the OTFT will degrade quickly. But the drain current values will exhibit a significant enhancement if 
we applied a proper bias voltage on the OTFT and the enhancement is reversible and reproducible. It shows the potential of being the memory device in the proper operation voltage based on this pentacene transistor [7].
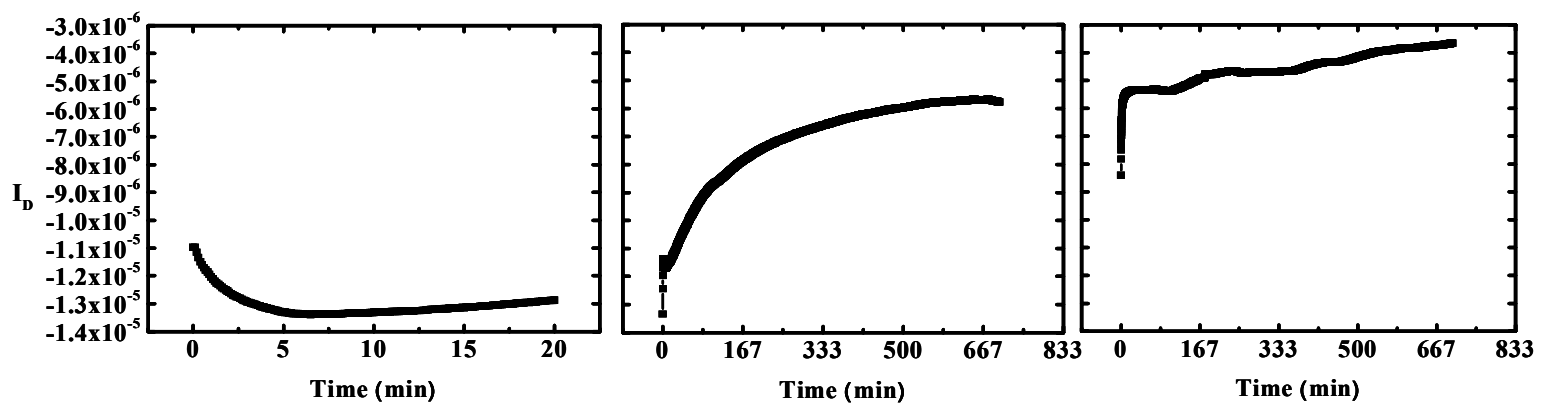

(a)
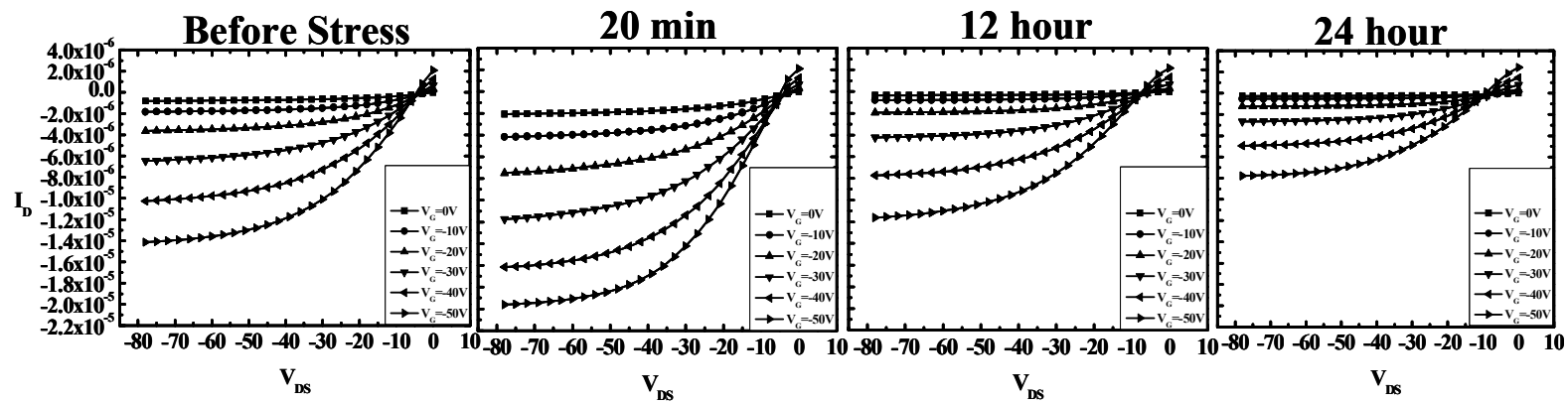

(b)

Fig. 5 (a) $\mathrm{I}_{\mathrm{D}}$ versus time under DC stress. (b) $\mathrm{I}_{\mathrm{d}}-\mathrm{V}_{\mathrm{ds}}$ curves of the corresponding stressing time.

Fig. 6 shows the more complicated measurement of bias stress. The definition of source and drain electrodes were exchanged after the bias stress. Then after another bias stress, the source and drain electrodes were changed back to the origin and the bias was applied again. In each times of stressing, we repeat the $\mathrm{I}_{\mathrm{d}}-\mathrm{V}_{\mathrm{ds}}$ measurements from 0 to $-50 \mathrm{~V}$ with the $\mathrm{V}_{\mathrm{g}}$ values of $0,-10,-20,-30,-40$, and $-50 \mathrm{~V}$ for 10 minutes. In Fig. $6(\mathrm{~b})$, the drain current was increased obviously from -0.75 to $-2.8 \mu \mathrm{A}$ at $\mathrm{V}_{\mathrm{g}}=-50 \mathrm{~V}$ and $\mathrm{V}_{\mathrm{ds}}=-50 \mathrm{~V}$. When the source and drain were exchanged, the current decreases to the $0.08 \mu \mathrm{A}$, nearly one tens of the original values, and it was almost recover back to the original values after another 10 minutes operation. It indicates that the holes were accumulated near the drain electrode after the bias stress, so the drain current was decreased significantly. Then after exchange the source and drain electrodes and applied the same bias operation, the carrier distribution was recovered to almost uniformly, result in the same current values as the original. In Fig. 6(e) and Fig. 6(f), the source and drain electrodes were change back to the origin and then another 10 minutes bias operation was applied, the increased of the drain current indicate that the operation is reproducible. 


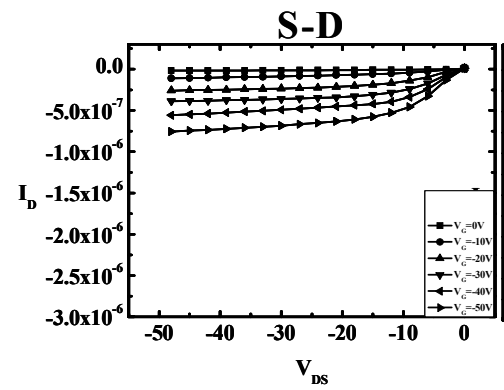

(a)

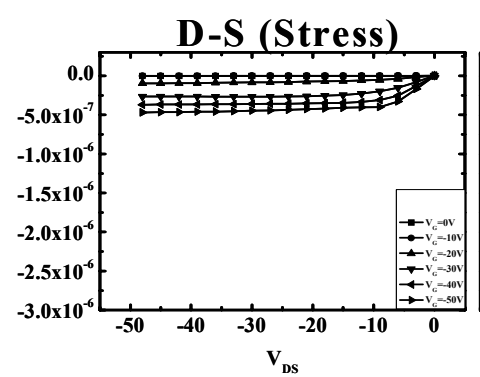

(d)

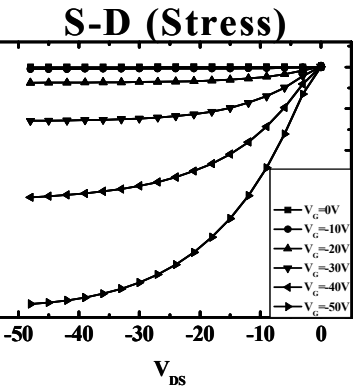

(b)

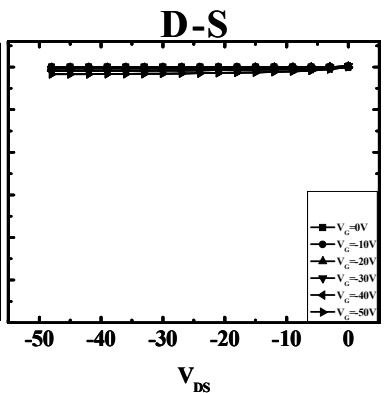

(c)

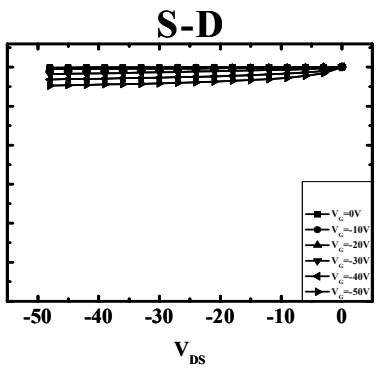

(e)

\section{S-D (Stress)}

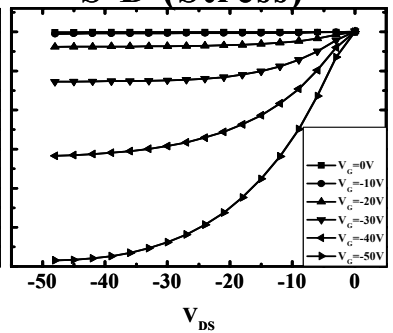

(f)

Fig. $6 \mathrm{I}_{\mathrm{d}}-\mathrm{V}_{\mathrm{ds}}$ curves of the corresponding stressing time. (a) before stressing (b) stressing for 10 minutes (c)exchange the source and drain electrodes (d)stressing for 10 minutes after exchange the source and drain electrodes (e) change back the source and drain electrodes (f) stressing for 10 minutes after change back the source and drain electrodes

\section{SUMMARY}

In summary, through the pretreatment of the surface, we have successfully fabricated the OTFT base on pentacene with high-mobility air-stable properties without heating the substrate during the evaporation. For the device with the channel length of $57 \mu \mathrm{m}$ and the channel width of $500 \mu \mathrm{m}$, the mobility value was $0.07 \mathrm{~cm}^{2} / \mathrm{Vs}$ and the degradation of the drain current was less than $30 \%$ after 12 days storage in the air environment. Besides, the increase of the drain current was observed after the bias stress. A 4 times increasing of the drain current values can be reached by repeating the $I_{d}-V_{d s}$ measurements from 0 to $-50 \mathrm{~V}$ with the $\mathrm{V}_{\mathrm{g}}$ values of $0,-10,-20,-30,-40$, and $-50 \mathrm{~V}$ for 10 minutes. This behavior is reversible and reproducible. It shows the potential of being a memory device under the proper bias voltage.

\section{ACKNOWLEDGMENTS}

This work was financially supported by ERSO/ITRI.

\section{REFERENCES}

1. G. Horowitz, Adv. Mater. 10, 365 (1998).

2. I. Kymissis, C. D. Dimitrakopoulos, and S. Purushothaman, IEEE Trans. on electron devices 48, 1060 (2001).

3. C. D. Dimitrakopoulos, and P. R. L. Malenfant, Adv. Mater. 14, 99 (2002).

4. Y. W. Wang, H. L. Cheng, Y. K. Wang, T. H. Hu, J. C. Ho, C. C. Lee, T. F. Lei, and C. F. Yeh, Thin Solid Films 467, 215 (2004).

5. S. J. Zilker, C. Detcheverry, E. Cantatore, and D. M. de Leeuw, Appl. Phys. Lett. 79, 1124 (2001).

6. M. Koehler, I. Biaggio, Phys. Rev. B 70, 045314 (2004).

7. G. Velu, C. Legrand, O. Tharaud, A. Chapoton, D. Remiens, G. Horowitz, Appl. Phys. Lett.79, 659 (2001). 